\title{
Assessment of laypersons' paediatric basic life support and foreign body airway obstruction management skills: a validity study
}

Asbjørn Hasselager ${ }^{1,2^{*}}$ (D), Doris Østergaard ${ }^{1,2}$, Tim Kristensen ${ }^{1,3}$, Claus Sønderskov ${ }^{4}$, Cathrine Bohnstedt ${ }^{5}$, Torsten L. B. Lauritsen ${ }^{6}$, Lars Konge ${ }^{2,7}$ and Martin G. Tolsgaard ${ }^{2,7}$

\begin{abstract}
Background: Standardised courses for laypeople in Paediatric Basic Life Support (PBLS) and Foreign Body Airway Obstruction Management (FBAOM) teach essential skills for the initiation of resuscitation by bystanders. Performance assessments are necessary to ensure that skills are acquired. We aimed to examine the validity of developed performance assessments and to determine credible pass/fail standards.

Methods: Validity evidence was gathered in a standardised simulated setting by testing participants with three different levels of PBLS/FBAOM experience: untrained laypersons, trained laypersons, and lifeguards. Two blinded raters assessed participants' performance. The reliability of test scores was analysed using generalizability theory, scores were compared across the three groups, and pass/fail-standards were established.

Results: A total of 33 participants were included. More than two raters and two cases were necessary for PBLS to achieve a reliability coefficient above 0.80 , which is considered the minimally acceptable level for high-stakes certification. For FBAOM, two tests or three raters were needed. Assessment scores differed across the three groups for PBLS skills, as well as for FBAOM skills $(p<0.001)$.

Pass levels of $74 \%$ and $55 \%$ of the maximum score for PBLS and FBAOM, respectively, were identified as the levels that best discriminated between competent and non-competent laypersons.

Conclusions: Laypersons' PBLS and FBAOM skills can be assessed in a reliable and valid way in a standardised simulated setting. However, multiple raters and scenario tests are needed to ensure sufficient reliability, which raises questions regarding the feasibility of performing certification tests for laypersons who participate in short paediatric resuscitation courses.
\end{abstract}

Keywords: Paediatric basic life support, Paediatric CPR, Laypersons, Foreign body airway obstruction, Training, Assessment, PBLS, FBAO, Validity, Pass/fail

\footnotetext{
* Correspondence: ahasselager@dadlnet.dk

${ }^{1}$ Copenhagen Academy for Medical Education and Simulation (CAMES) Herlev Ringvej 75, 2730 Herlev, Denmark

${ }^{2}$ University of Copenhagen, Nørregade 10, 101 Copenhagen, Denmark

Full list of author information is available at the end of the article
}

(c) The Author(s). 2018 Open Access This article is distributed under the terms of the Creative Commons Attribution 4.0 International License (http://creativecommons.org/licenses/by/4.0/), which permits unrestricted use, distribution, and reproduction in any medium, provided you give appropriate credit to the original author(s) and the source, provide a link to the Creative Commons license, and indicate if changes were made. The Creative Commons Public Domain Dedication waiver (http://creativecommons.org/publicdomain/zero/1.0/) applies to the data made available in this article, unless otherwise stated. 


\section{Background}

Survival from out-of-hospital paediatric cardiac arrest depends on fast recognition and initiation of resuscitation by bystanders [1-3]. To increase paediatric survival, relevant target groups, including daycare employees and other non-medical personnel working with children, need to possess resuscitation skills. Standardised courses for laypeople in Paediatric Basic Life Support (PBLS) and Foreign Body Airway Obstruction Management (FBAOM) are designed to teach the necessary skills based on international guidelines [4]. However, assessments are needed to ensure that course participants have acquired the skills necessary to deliver effective PBLS and FBAOM in the future.

Existing assessment instruments for paediatric resuscitation skills are directed at highly skilled health professionals who work in an in-hospital setting [5-7]. Effective first response intervention requires less advanced skills, than those expected in-hospital and can be taught to laypersons with no pre-existing medical training. Previous studies have used assessment instruments adapted from guidelines or extrapolated from existing assessment instruments designed for resuscitation of adults to determine readiness for practice [8-11]. However, such assessments may not be valid markers of competence when used for different populations, skills, and purposes [12].

Assessment of laypersons' PBLS and FBAOM skills should have established validity evidence to support the interpretations made based on the assessment scores (i.e. is this person able to deliver effective PBLS/FBAOM?). In a recent study, essential items for the assessment of the two lifesaving skills, PBLS and FBAOM, were identified in an international consensus study [13]. However, evidence supporting the interpretation of test scores based on these items needs to be established. Without established validity evidence the value of assessments for both formative (e.g. assessment for feedback) and summative purposes (e.g. assessment for certification) is limited [14-16].

The objectives of this study were to collect validity evidence for the assessment of laypersons' PBLS and FBAOM skills and to establish credible pass/fail standards.

\section{Methods}

\section{Study design and setting}

The study was conducted in a simulated setting in Copenhagen, Denmark and enrolled 33 laypersons between March and June 2017.

The study was deemed exempt from ethics approval by the Ethical Committee of the Capital Region, Copenhagen Denmark (Protocol no. 17006007). The Danish Data Protection Agency approved the study (j.nr: 2012-58-0004). All participants provided informed consent prior to enrolment in the study.
Messick's framework for validity evidence was used in this study and is recommended by the American Education Research Association and the American Psychological Association in the 2014 Standards for Educational Testing [17]. The framework includes five categories of evidence: content, response process, internal structure, relation to other variables, and consequences [12]. A flowchart depicting the categories and the study design used to collect evidence is available in the appendix (Additional file 1 - Appendix figure 1).

\section{Participants}

Purposive and convenience sampling was strategically performed to include three different groups: untrained laypersons, laypersons trained on PBLS and FBAOM, and lifeguards.

The three participant groups included in this study represented different levels of PBLS/FBAOM experience and were expected to have increasing levels of PBLS/FBAOM skills.

The untrained laypersons were daycare employees with no resuscitation training in the past year.

The trained laypersons group consisted of daycare employees, who participated in a two-hour hands-on standardised instructor-led course with up to six participants, immediately prior to the scenario tests. The course involved focused training on child and infant PBLS and FBAOM skills following ERC guidelines [4] and used the same manikins as the PBLS and FBAOM scenario tests. Instructors were basic life support certified instructors with additional paediatric training.

Lifeguards participated in a three-day intensive course just prior to the scenario tests. The course involved general first aid and basic life support provider resuscitation training with additional resuscitation training for children and infants.

Exclusion criteria for untrained and trained laypersons were any first aid training within one year, any type of health professional education. Skills generally decay over as little as six months and we chose a minimum of one year to avoid influence from previous training [7].

\section{Performance tests}

The participants conducted two standardised simulated scenario tests for PBLS and FBAOM, respectively (Fig. 1).

Prior to the testing, participants were introduced to the simulated environment and informed about the purpose of the tests. A test facilitator led the scenarios using a standardised instruction protocol.

The PBLS scenario test included a child who was found lifeless on the floor in a daycare. The participant was alone at the scene and a helper was present elsewhere in the daycare centre. The PBLS test was conducted using Little Junior ${ }^{\mathrm{rux}}$ manikins (Laerdal Medical, Stavanger, Norway). The FBAOM test scenario involved an infant with sudden foreign body airway obstruction 


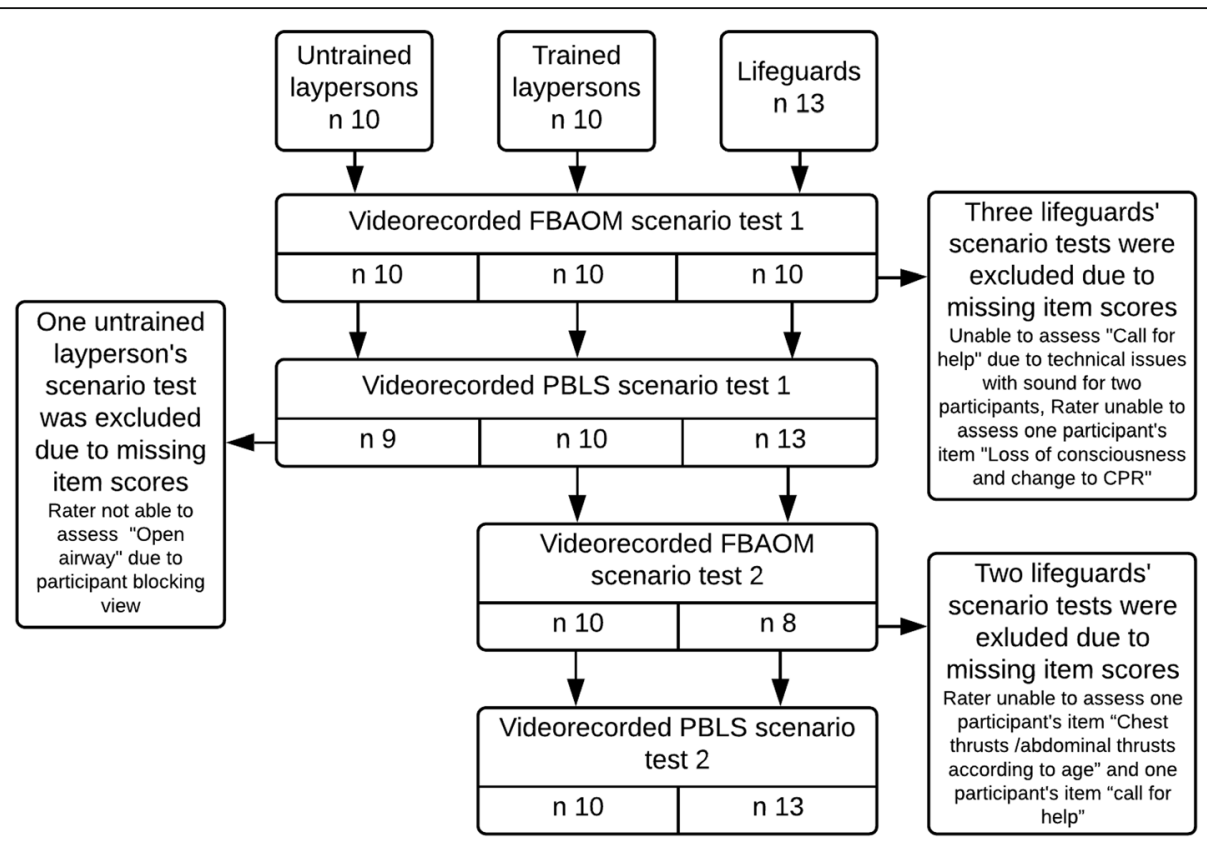

Fig. 1 Flowchart for tests by participants. Legend to Fig. 1: The flowchart illustrates the participants in the scenario tests by group and exclusions due to missing rater scorings

with rapid deterioration into unconsciousness. The Baby Anne $^{\mathrm{Tx}}$ manikin (Laerdal Medical, Stavanger, Norway) was used for the FBAOM tests. The scenario context was explained to the participants: E.g. "You are alone in a daycare centre with a ten month old child who suddenly gets something stuck in the throat. The child is coughing loudly, awake and crying. There is no one else nearby. Show what you would do."

The scenario tests were repeated once with slight alterations in the child's age and circumstances (Fig. 1). The clinical problem was identical for the two repeated tests and the expected actions according to current guidelines were the same.

Each test had a duration of approximately two to five minutes. The tests were video-recorded and viewed using iPads ${ }^{\text {tw }}$ (Apple, California, USA).

The content of the PBLS and FBAOM assessment instruments was determined in an international Delphi consensus study which identified which elements should be included in assessments of laypersons [13]. The instruments included nine items for PBLS and eight for FBAOM. One item for PBLS "Use of AED" was not applicable for the training of the layperson group and hence excluded. Each assessment item was evaluated based on five-point scales. The research group developed descriptive anchors for values one, three and five, which targeted expectations for laypersons. The authors discussed the descriptive anchors until consensus was achieved.

Five-point scales were used instead of checklists to better capture increasing levels of competence [18].
The resulting assessment instruments for PBLS and FBAOM are shown in the appendix (Additional file 1 Appendix tables 1 and 2).

A pilot test revealed that four out of eight FBAOM items could be assessed based on video-recorded scenario tests, and that for one FBAOM item ("Identify loss of consciousness and change to CPR") only part of the original item could be assessed. The ability to identify unconsciousness was not possible to assess due to the limitations imposed by the manikin, and consequently, only the participant's actions in response to unconsciousness were assessed.

The individual item scores were added to generate an assessment score. The maximum score for the two instruments were 40 and 20 points for PBLS and FBAOM, respectively. In addition to the item scores, the scenario tests were assessed using a 7-point global rating scale for the participant's performance $(1=$ poor $-7=$ excellent $)$.

The response process included assessment of the scenario test videos in a random order by two blinded raters, who were European Resuscitation Council (ERC) certified BLS instructors. The raters participated in a 5-h rater-training course prior to rating the scenario tests. During the rater-training course, pilot rating videos were assessed and discussed with raters until consensus was reached.

\section{Statistics}

The internal structure was examined by Generalizability (G) theory to examine the variances that influenced the reliability of the PBLS and FBAOM assessment scores. 
G theory allows analysis of all the sources of variance (facets) and their interactions at the same time, such as interrater and test-retest variance, and enables the prediction of how test reliability changes when facet conditions are changed [19]. $G$ theory is recommended for producing reliability estimates when assessing procedural skills [20].

The assessment scores of trained laypersons and lifeguards by each of the two raters were analysed separately for FBAOM and PBLS. The analysis was done using the G1 G theory program for SPSS [21]. Untrained laypersons were not included, as they are not the intended target population for the assessment instruments, and would, therefore, overinflate the reliability coefficients without reflecting the test's intended use [22]. We used a fully-crossed two-facet design, with raters and tests as facets to estimate variances from these sources.

The variance attributed to the participants was considered the true variance reflecting different levels of competence. Error contributions were variances that related to raters and tests, as well as interactions with these. The percentage of the total variance was calculated to explain the true score fraction of the PBLS and FBAOM scores, respectively. Subsequently, the variance components were used in a decision-study (d-study) to determine the number of tests and raters needed to provide reliable judgments. A G coefficient of 0.8 is generally considered sufficient for high-stakes exams and 0.6 sufficient for formative feedback [19].

Internal consistency was examined using Cronbach's alpha for the PBLS and FBAOM assessment instrument items, separately. Correlations of assessment instrument scores and global rating scores were analysed using Pearson's correlation coefficients.

The relationship to other variables was examined by group comparisons. Assessment scores were the mean of the two raters' scores as a percentage of maximum score. The assessment scores were compared using one-way analysis of variances (ANOVA) across the three groups and Bonferroni post hoc analysis between groups to examine their abilities to discriminate between different levels of skill. Only the assessment scores for the first scenario test for PBLS and FBAOM were included to avoid a testing effect [23].

The consequences were examined by the contrasting groups' method to determine a pass/fail level based on the distribution of mean scores for untrained laypersons and lifeguards [24].

The intersection of the score distribution for the two groups indicated the level which ensures as few false negatives (failing competent performers - lifeguards) and false positives (passing incompetent performers - untrained laypersons) as possible. The contrasting groups' pass/fail level and theoretical false positive and false negative distributions were calculated using a previously published Excel code [24].
SPSS version 24 was used for all other statistical analyses. A significance level of 0.05 was used for all analyses.

\section{Results}

Characteristics of the participants are shown in Table 1. There were six missing assessment scores out of a total of 112 possible assessment scores (Fig. 1).

Table 2 demonstrates results from the validation process structured according to Messick's five sources of validity evidence.

The generalizability analysis is shown in appendix (Additional file 1 - Appendix table 4). The d-study results are shown in Fig. 2. The d-study demonstrated that three raters and three cases or one rater and six cases were needed to achieve a reliability coefficient of 0.80 for PBLS. For FBAOM, three raters or two tests were needed. The Cronbach's alpha was 0.94 and 0.64 for PBLS and FBAOM assessment item scores, respectively. Pearson's correlation coefficients between the assessment scores and the global rating scores were $\mathrm{r}(30)=0.93, p<0.001$ for PBLS and $\mathrm{r}(28)=0.96, \mathrm{p}<0.001$ for FBAOM.

PBLS and FBAOM assessment scores differed significantly across the three groups for both PBLS $(F(2,29)=$ 64.01, $\mathrm{p}<0.001)$ and FBAOM $(\mathrm{F}(2,27)=13.04, \mathrm{p}<$ 0.001 ). Mean scores and post-hoc analysis are shown in Table 3.

The individual item scores and analysis are presented in the appendix (Additional file 1 - Individual item scores).

The pass/fail level was established as $74 \%$ and $55 \%$ of the maximum score for PBLS and FBAOM, respectively (Fig. 3). All the untrained laypersons, $20 \%$ of the trained laypersons and $8 \%$ of the lifeguards failed the PBLS scenario test. For FBAOM, $80 \%$ of the untrained laypersons, none of the trained laypersons and $30 \%$ of the lifeguards failed.

\section{Discussion}

The validity evidence supports the assumption that increasing scores reflect increasing levels of PBLS and

Table 1 Characteristics of participants

\begin{tabular}{llll}
\hline & $\begin{array}{l}\text { Untrained } \\
\text { laypersons }\end{array}$ & $\begin{array}{l}\text { Trained } \\
\text { laypersons }\end{array}$ & Lifeguards \\
\hline Participants $(\mathrm{n})$ & 10 & 10 & 13 \\
Sex, female & $10(100)$ & $10(100)$ & $9(69)$ \\
Age, years & $40(34-46)$ & $49(35-53)$ & $20(20-21)$ \\
Working with children, Years & $10(3-12)$ & $11(7-25)$ & - \\
No prior training & $3(30)$ & $3(30)$ & - \\
Years since training & & & \\
$2-5$ & $1(10)$ & $3(30)$ & - \\
$>5$ & $6(60)$ & $4(40)$ & - \\
\hline
\end{tabular}

Continuous data are reported as median (Interquartile range). Dichotomous data reported as $\mathrm{n}(\%)$ 
Table 2 Validity evidence results by category

\begin{tabular}{|c|c|c|c|}
\hline \multirow{2}{*}{$\begin{array}{l}\text { Validity evidence } \\
\text { source }\end{array}$} & \multirow{2}{*}{$\begin{array}{l}\text { Question related to the } \\
\text { source of evidence }\end{array}$} & \multicolumn{2}{|l|}{ Validity evidence for the assessment } \\
\hline & & PBLS & FBAOM \\
\hline Content & $\begin{array}{l}\text { Is the content measuring } \\
\text { the intended construct } \\
\text { (skill levels of laypersons)? }\end{array}$ & \multicolumn{2}{|c|}{ International resuscitation experts identified the assessment items as essential for laypersons } \\
\hline \multirow[t]{2}{*}{$\begin{array}{l}\text { Response } \\
\text { process }\end{array}$} & \multirow[t]{2}{*}{ Are bias sources reduced? } & $\begin{array}{l}\text { One item was not applicable to the } \\
\text { layperson training and excluded. }\end{array}$ & $\begin{array}{l}\text { Pilot testing of the rating procedure revealed } \\
4 \text { FBAOM items could not be scored. }\end{array}$ \\
\hline & & \multicolumn{2}{|c|}{ The raters participated in rater training and participants' skill levels were blinded for the raters. } \\
\hline \multirow[t]{3}{*}{$\begin{array}{l}\text { Internal } \\
\text { structure }\end{array}$} & \multirow[t]{3}{*}{$\begin{array}{l}\text { Are the test scores } \\
\text { reliable? }\end{array}$} & \multicolumn{2}{|c|}{$\begin{array}{l}\text { The generalizability analysis and the d-study identified the number of tests and raters needed } \\
\text { for different levels of reliability. }\end{array}$} \\
\hline & & \multicolumn{2}{|c|}{$\begin{array}{l}\text { Pearson's correlations above } 0.93(p<0.001) \text { between global ratings scores and assessment scores } \\
\text { support the construct of the test. }\end{array}$} \\
\hline & & $\begin{array}{l}\text { The high Cronbach's alpha supports the } \\
\text { match of items and the intended construct }\end{array}$ & $\begin{array}{l}\text { The questionable Cronbach's alpha suggests } \\
\text { internal inconsistency in the test items. }\end{array}$ \\
\hline $\begin{array}{l}\text { Relation to } \\
\text { other variables }\end{array}$ & $\begin{array}{l}\text { Does the score } \\
\text { correlate with } \\
\text { other measures } \\
\text { of skills? }\end{array}$ & $\begin{array}{l}\text { The assessment scores increased with } \\
\text { increasing duration of training and } \\
\text { significantly differentiated all the } \\
\text { three groups. }\end{array}$ & $\begin{array}{l}\text { The assessment scores increased with training and } \\
\text { discriminated untrained laypersons from all other groups } \\
\text { The assessment scores were not able to discriminate } \\
\text { trained laypersons from lifeguards. }\end{array}$ \\
\hline \multirow[t]{2}{*}{ Consequences } & $\begin{array}{l}\text { What is the consequences } \\
\text { of the pass/fail score }\end{array}$ & $\begin{array}{l}\text { All untrained laypersons and one lifeguard } \\
\text { failed. Theoretical false positives and } \\
\text { negative with the contrasting groups } \\
\text { method was } 1.0 \% \text { and } 0.5 \% \text {, respectively. }\end{array}$ & $\begin{array}{l}\text { Eight untrained laypersons and three lifeguards failed. } \\
\text { Theoretical false positives and negative with the } \\
\text { contrasting groups method was } 22 \% \text { and } 29 \% \text {, } \\
\text { respectively }\end{array}$ \\
\hline & & \multicolumn{2}{|c|}{$\begin{array}{l}\text { Unintended consequences of the pass score could be low self-efficacy and reluctance to intervene } \\
\text { in real resuscitation attempts }\end{array}$} \\
\hline
\end{tabular}

The table shows the five categories and the validity evidence in each category

FBAOM skills. The PBLS and FBAOM assessment scores significantly discriminated untrained from trained laypersons and lifeguards (Table 3). The validity argument apparent in our findings is further supported by the strong correlations between PBLS/FBAOM assessment scores and the global rating scores.

The PBLS d-study (Fig. 2) shows that two tests or two raters are needed to reach $G$ coefficients of 0.6 which are sufficient for formative feedback, and six tests for one rater or three tests and two raters are needed for high stakes certification $G$ coefficients of 0.8 . For FBAOM (Fig. 2), a $\mathrm{G}$ coefficient of 0.6 requires one test and one rater, and a $\mathrm{G}$ coefficient of 0.8 requires at least two tests or three raters.

A generalizability analysis for residents' advanced paediatric life support skills found similar results such that additional tests increased reliability more than additional raters [5]. In fact, 12 tests were needed for a generalizability coefficient of 0.73 , and another study with ten tests and two raters resulted in a $\mathrm{G}$ coefficient of 0.94 [6].

The results of our d-study reflect the need for fewer tests to reach sufficient reliability. This may be because our scenario tests were less specialised, and test the same skills in each scenario test, as illustrated by the very low variance contribution from tests in the g study (Additional file 1 - Appendix table 4).

Certification of layperson may not be feasible within the short duration of traditional PBLS courses without compromising the time dedicated to actual PBLS training. However, reliability coefficients sufficient for formative feedback to improve learning may be achievable for both PBLS and FBAOM [16]. In addition, the process of testing individuals could also, by itself, induce a learning effect [23].

FBAOM assessment scores revealed that the lifeguards, who were expected to perform at the highest level, were matched by trained laypersons (Table 3). The trained laypersons participated in specific FBAOM training just prior to the scenario test. In addition, the infant FBAOM skills may be mostly relevant for daycare employees which may increase motivation among laypersons to learn these skills, whereas the lifeguards may be more focused on skills that they are expected to master, such as FBAOM for adults and general resuscitation skills. The findings are similar to a previous assessment of residents in paediatric advanced life support, where experience did not affect performance, but specific training improved all residents' performance [6]. An alternative explanation is that the assessment instrument was not able to capture experts' skills, which may rely on shortcuts and less strict adherence to a step-by-step approach than the approaches of untrained laypersons [25]. However, the high correlation with the overall performance score of 0.96 suggests that this was not the case.

For PBLS, the pass/fail level of $74 \%$ clearly discriminated competent from non-competent performers and 


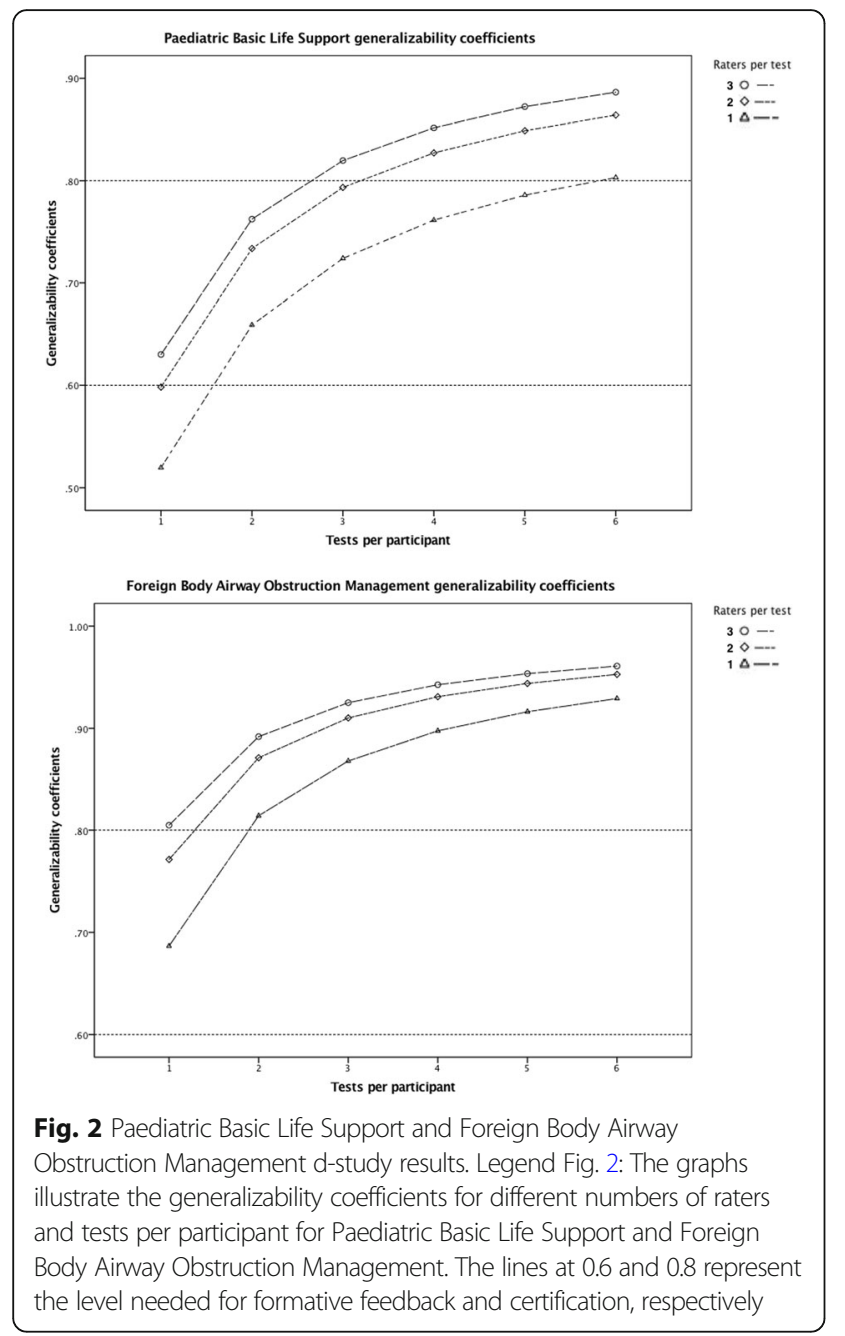

the theoretical distributions revealed only $1.0 \%$ false positives (passing incompetent performers) and $0.5 \%$ false negatives (failing competent performers) (Fig. 3).

For FBAOM, the pass/fail level was 55\% and the theoretical distribution of scores resulted in $22 \%$ false positives (passing incompetent performers) and 29\% false negatives (failing competent performers) (Fig. 3).

Most untrained laypersons can attain sufficient skill levels with short standardised training for both PBLS and FBAOM (Fig. 3). Performance improvements has

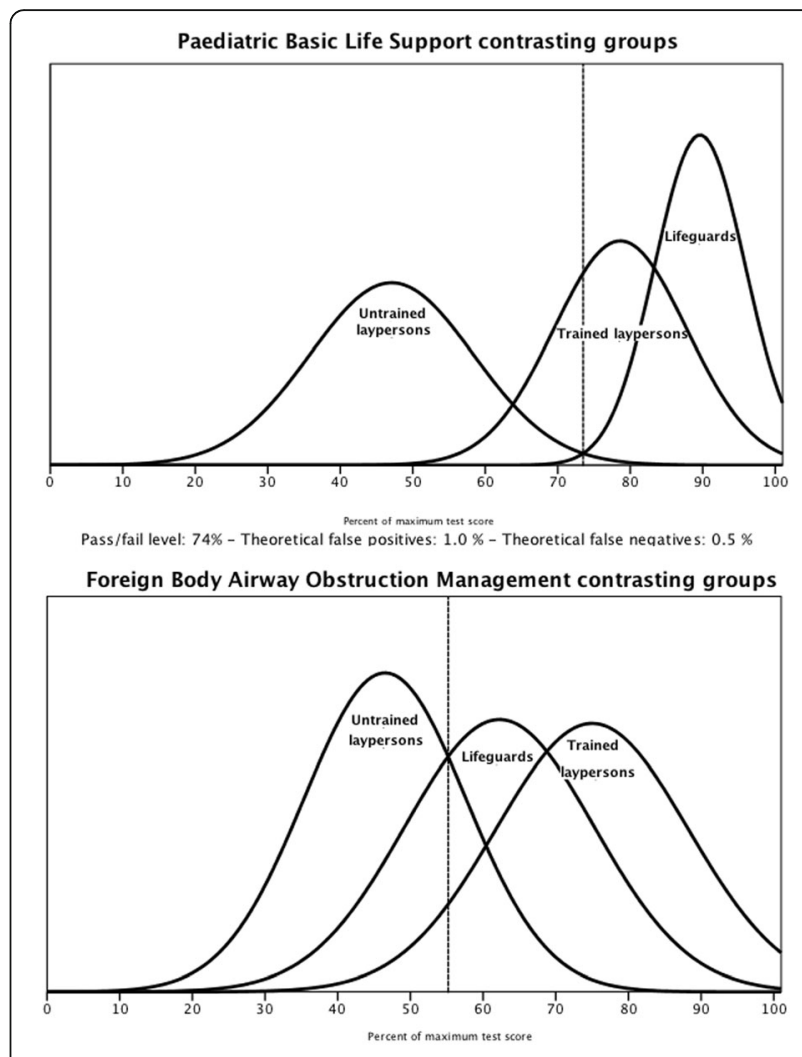

Pass/fail level: $55 \%$ - Theoretical false positives: $22 \%$ - Theoretical false negatives: $29 \%$

Fig. 3 Paediatric Basic Life Support and Foreign Body Airway Obstruction Management contrasting groups. Legend Fig. 3: The figures illustrate the distributions of the three groups and the pass/fail level for Paediatric Basic Life Support and Foreign Body Airway Obstruction Management based on the intersection between the untrained laypersons' and lifeguards' distributions. The theoretical distributions of false positives and false negatives are displayed. The $Y$ axis illustrates the relative number of participants receiving each score

also been demonstrated for laypersons who receive brief training in adult resuscitation skills [26, 27].

However, the pass/fail level for FBAOM allows a large proportion of non-competent performers to receive a passing score. Hence, the level may not be advisable for the purpose of certification, particularly given the low reliability if only a single test and a single rater are used. Moreover, there may be unintended consequences of failing some course participants with respect to reduced

Table 3 Assessment score means and post hoc analysis

\begin{tabular}{|c|c|c|c|c|c|c|c|}
\hline & \multirow{2}{*}{$\begin{array}{l}\text { Untrained laypersons, } \\
\text { mean }(95 \% \mathrm{Cl})\end{array}$} & \multirow{2}{*}{$\begin{array}{l}\text { Trained laypersons, } \\
\text { mean }(95 \% \mathrm{Cl})\end{array}$} & \multirow{2}{*}{$\begin{array}{l}\text { Lifeguards, } \\
\text { mean }(95 \% \mathrm{Cl})\end{array}$} & \multirow{2}{*}{$\begin{array}{l}\text { One-way- } \\
\text { ANOVA }\end{array}$} & \multicolumn{3}{|c|}{ Post hoc analysis (t-test with Bonferroni corrected $p$-value*) } \\
\hline & & & & & $\begin{array}{l}\text { Untrained laypersons } \\
\text { vs. Trained laypersons }\end{array}$ & $\begin{array}{l}\text { Untrained } \\
\text { laypersons } \\
\text { vs. lifeguards }\end{array}$ & $\begin{array}{l}\text { Trained } \\
\text { laypersons } \\
\text { vs. lifeguards }\end{array}$ \\
\hline PBLS & $\begin{array}{l}47.08 \\
(38.41-55.76)\end{array}$ & $\begin{array}{l}78.63 \\
(72.06-85.19)\end{array}$ & $\begin{array}{l}89.62 \\
(85.85-93.38)\end{array}$ & $\begin{array}{l}F(2,29)=64.011 \\
p<0.001)\end{array}$ & $\begin{array}{l}\mathrm{t}(18)=-5.21 \\
p<0.001\end{array}$ & $\begin{array}{l}\mathrm{t}(20)=-11.38 \\
p<0.001\end{array}$ & $\begin{array}{l}\mathrm{t}(21)=-3.42 \\
p=0.02\end{array}$ \\
\hline FBAOM & $\begin{array}{l}46.50 \\
(38.54-54.46)\end{array}$ & $\begin{array}{l}75.00 \\
(65.54-84.46)\end{array}$ & $\begin{array}{l}62.25 \\
(52.92-71.58)\end{array}$ & $\begin{array}{l}F(2,27)=13.038 \\
p<0.001\end{array}$ & $\begin{array}{l}\mathrm{t}(17)=-6.72 \\
p<0.001\end{array}$ & $\begin{array}{l}\mathrm{t}(18)=-2.91 \\
p=0.03\end{array}$ & $\begin{array}{l}\mathrm{t}(18)=2.17 \\
p=0.09\end{array}$ \\
\hline
\end{tabular}

The table shows the ANOVA of assessment mean scores as per cent of max score for PBLS and FBAOM and the Bonferroni adjusted post hoc analysis. *Values $<0.05$ are considered significant 
self-efficacy and willingness to initiate real resuscitation attempts, which in turn, may reduce the chance of survival $[1-3]$. On the other hand, passing a course implies that participants have attained certain skills which can be used to provide effective resuscitation attempts.

The reliability results are strengthened by inclusion of only trained laypersons and lifeguards in the generalizability analysis, as reliability indices will be artificially overinflated by including complete untrained in the calculation [22, 28].

A limitation to the study is the number of participants, although the sample size was larger than the median sample size $(n=25)$ of education research studies [29], and significant differences were identified between groups.

We used convenience sampling which may have resulted in selection of participants who were more motivated about training than the general population. In turn, this may have resulted in better performance among untrained and trained laypersons. However, we believe that most daycare workers are motivated about gaining paediatric resuscitation skills.

Internal consistency of the FBAOM test was questionable (Cronbach's alpha $=0.64)$. One item "call for help" seemed to be problematic (Additional file 1 - Appendix table 3). The item failed to discriminate between groups $(F(2,28)=2.27$, $p=0.12$ ) and omitting it from the FBAOM assessment instrument may be advisable from a psychometric point of view, as it does not help to discriminate between the three groups of performers. However, content experts considered this item essential for the assessment [13] and it is still a vital part of the chain of survival [30]. For these reasons, we chose to retain the item, as we suspect that the poor fit in our study reflects failure to assess participants' ability to call for help in the simulated setting rather than that the item is non-essential.

The primary implication of the study is that the PBLS and FBAOM assessment instruments can be used to assess laypersons' PBLS and FBAOM skill levels. The assessment scores make it possible to compare outcomes from different training methods and to assess the quality of various courses. Moreover, the use of standardised performance standards enables competency-based training as an alternative to current time-based models.

The reliability analyses suggest that the assessment instruments can be used for formative feedback to increase learning for laypersons, but not for summative certification purposes if only one or two tests administered. However, if certification of laypersons skills is needed courses should be designed with additional time to allow for an appropriate number of tests and raters for defensible certification of skill levels.

\section{Conclusions}

The study found evidence to support the use of standardised assessment instruments to measure increasing skill levels in PBLS and FBAOM.
Reliable assessments of performance for formative feedback purposes are attainable. However, multiple raters and scenario tests are needed to ensure reliability which is sufficient to justify PBLS and FBAOM certification, and this may not be feasible during brief training courses for laypersons.

\section{Additional file}

Additional file 1: The appendix includes an overview of the scoring instruments, flowchart for collecting validity evidence, analysis of individual item scores and results of the generalizability analysis. (PDF $626 \mathrm{~kb}$ )

\section{Abbreviations}

ERC: European Resuscitation Council; FBAOM: Foreign Body Airway Obstruction Management; PBLS: Paediatric Basic Life Support

\section{Availability of data and materials}

The data that support the findings of this study are available on request from the corresponding author $\mathrm{AH}$, The data are not publicly available due to them containing information that could compromise participant privacy/consent.

\section{Funding}

Asbjørn Hasselager received unrestricted grants from TrygFonden (ID: 116660) and Laerdal Foundation (no 3253).

\section{Authors' contributions}

$\mathrm{AH}$ gathered data and prepared the first draft. All authors have made substantial contributions to the following: (1) the conception and design of the study, or acquisition of data, or analysis and interpretation of data, (2) drafting the article or revising it critically for important intellectual content, (3) final approval of the version to be submitted. All authors read and approved the final manuscript.

Ethics approval and consent to participate

The study was deemed exempt from ethics approval by the Ethical Committee of the Capital Region, Copenhagen Denmark (Protocol no. 17006007). The Danish Data Protection Agency approved the study (j.nr: 2012-58-0004). All participants provided informed consent prior to enrolment in the study.

\section{Consent for publication}

Not applicable.

\section{Competing interests}

The authors declare that they have no competing interests.

\section{Publisher's Note}

Springer Nature remains neutral with regard to jurisdictional claims in published maps and institutional affiliations.

\footnotetext{
Author details

${ }^{1}$ Copenhagen Academy for Medical Education and Simulation (CAMES), Herlev Ringvej 75, 2730 Herlev, Denmark. '2University of Copenhagen, Nørregade 10, 101 Copenhagen, Denmark. ${ }^{3}$ Department of Children and Adolescence Medicine, Herlev Hospital, Herlev Ringvej 75, 2730 Herlev, Denmark. ${ }^{4}$ RedMitBarn - FirstAiders, Rosenørns Alle 1, 1970 Frederiksberg C, Denmark. ${ }^{5}$ Department of paediatrics, North Zealand Hospital, Dyrehavevej 29, 3400 Hillerød, Denmark. ${ }^{6}$ Department of Anaesthesia, The Juliane Marie Centre, Rigshospitalet University Hospital, Blegdamsvej 9, 2100 Copenhagen, Denmark. ${ }^{7}$ Copenhagen Academy for Medical Education and Simulation (CAMES), Blegdamsvej 9, 2100 Copenhagen, Denmark.
} 
Received: 19 June 2018 Accepted: 31 August 2018

Published online: 06 September 2018

\section{References}

1. Kitamura T, Iwami T, Kawamura T, Nagao K, Tanaka H, Nadkarni VM, et al. Conventional and chest-compression-only cardiopulmonary resuscitation by bystanders for children who have out-of-hospital cardiac arrests: a prospective, nationwide, population-based cohort study. Lancet. 2010;375:1347-54.

2. Naim MY, Burke RV, McNally BF, Song L, Griffis HM, Berg RA, et al. Association of Bystander Cardiopulmonary Resuscitation with Overall and Neurologically Favorable Survival after Pediatric out-of-Hospital Cardiac Arrest in the United States: a report from the cardiac arrest registry to enhance survival surveillance registry. JAMA Pediatr. 2017:171:133-41.

3. Goto Y, Maeda T, Goto Y. Impact of dispatcher-assisted bystander cardiopulmonary resuscitation on neurological outcomes in children with out-of-hospital cardiac arrests: a prospective, nationwide, population-based cohort study. J Am Heart Assoc. 2014;3:e000499-9.

4. Maconochie IK, Bingham R, Eich C, López-Herce J, Rodríguez-Núñez A, Rajka T, et al. European resuscitation council guidelines for resuscitation 2015: section 6. Paediatric life support. Resuscitation. 2015:95:223-48.

5. Donoghue A, Nishisaki A, Sutton R, Hales R, Boulet J. Reliability and validity of a scoring instrument for clinical performance during pediatric advanced life support simulation scenarios. Resuscitation. 2010;81:331-6.

6. Levy A, Donoghue A, Bailey B, Thompson N, Jamoulle O, Gagnon R, et al. External validation of scoring instruments for evaluating pediatric resuscitation. Simul Healthc. 2014:9:360-9.

7. Greif R, Lockey AS, Conaghan P, Lippert A, De Vries W, Monsieurs KG, et al. European resuscitation council guidelines for resuscitation 2015: section 10 Education and implementation of resuscitation. Resuscitation. 2015;95:288-301.

8. Shavit I, Peled S, Steiner IP, Harley DD, Ross S, Tal-Or E, et al. Comparison of outcomes of two skills-teaching methods on lay-rescuers' acquisition of infant basic life support skills. Acad Emerg Med. 2010;17:979-86.

9. Krogh LQ, Bjørnshave K, Vestergaard LD, Sharma MB, Rasmussen SE, Nielsen $\mathrm{HV}$, et al. E-learning in pediatric basic life support: a randomized controlled non-inferiority study. Resuscitation. 2015;90C:7-12.

10. Hawkes GA, Murphy G, Dempsey EM, Ryan AC. Randomised controlled trial of a mobile phone infant resuscitation guide. J Paediatr Child Health. 2015: 51:1084-8.

11. Peters M, Stipulante S, Delfosse A-S, Schumacher K, Mulder A, Lebrun F, et al. Dispatcher-assisted telephone cardiopulmonary resuscitation using a French-language compression-ventilation pediatric protocol. Pediatr Emerg Care. 2017;33:679-85.

12. Cook DA, Beckman TJ. Current concepts in validity and reliability for psychometric instruments: theory and application. Am J Med. 2006;119:166.e7-16.

13. Hasselager AB, Lauritsen T, Kristensen T, Bohnstedt C, Sønderskov C, Ostergaard $D$, et al. What should be included in the assessment of laypersons' paediatric basic life support skills? Results from a Delphi consensus study. Scand J Trauma Resusc Emerg Med. 2018:26:9.

14. Cook DA, Zendejas B, Hamstra SJ, Hatala R, Brydges R. What counts as validity evidence? Examples and prevalence in a systematic review of simulationbased assessment. Adv Health Sci Educ Theory Pract. 2014;19:233-50.

15. Van Der Vleuten C, Sluijsmans D, Joosten-ten Brinke D. Competence Assessment as Learner Support in Education. Competence-based Vocationa and Professional Education. 5 ed. Cham: Springer International Publishing; 2016. p. 607-30.

16. Bhanji F, Donoghue AJ, Wolff MS, Flores GE, Halamek LP, Berman JM, et al. Part 14: education: 2015 American Heart Association guidelines update for cardiopulmonary resuscitation and emergency cardiovascular care. Circulation. 2015:S561-73.

17. Association AER, Association AP. National Council on measurement in education., educational JCOSF, S PTU. Standards for educational and psychological testing. 2014 edition. Washington, DC: American Educational Research Association; 2014

18. Ilgen JS, Ma IWY, Hatala R, Cook DA. A systematic review of validity evidence for checklists versus global rating scales in simulation-based assessment. Med Educ. 2015;49:161-73.

19. Bloch R, Norman G. Generalizability theory for the perplexed: a practical introduction and guide: AMEE guide no. 68. Med Teach. 2012;34:960-92.

20. Ahmed K, Miskovic D, Darzi A, Athanasiou T, Hanna GB. Observational tools for assessment of procedural skills: a systematic review. Am J Surg. 2011;202: 469-480.e6.
21. Mushquash C, O'Connor BP. SPSS and SAS programs for generalizability theory analyses. Behav Res Methods. 2006;38:542-7.

22. Cook DA. Much ado about differences: why expert-novice comparisons add little to the validity argument. Adv in Health Sci Educ. 2014;20:829-34.

23. Kromann CB, Jensen ML, Ringsted C. The effect of testing on skills learning. Med Educ. 2009:43(1):21-7.

24. Jørgensen $M$, Konge $L$, Subhi $Y$. Contrasting groups' standard setting for consequences analysis in validity studies: reporting considerations. Adv Simul. 2018;3:5

25. Hodges B, Regehr G, McNaughton N, Tiberius R, Hanson M. OSCE checklists do not capture increasing levels of expertise. Acad Med. 1999;74:1129-34.

26. Berden HJ, Pijls NH, Willems FF, Hendrick JM, Crul JF. A scoring system for basic cardiac life support skills in training situations. Resuscitation. 1992;23: $21-31$.

27. Sim MS, Jo IJ, Song HG. Basic cardiac life support education for non-medical hospital employees. Emerg Med J. 2009;26:327-30.

28. Streiner DL, Norman GR. Reliability. In: Health measurement scales a practical guide to their development and use. New York: Oxford University Press; 2008. p. 167-210.

29. Cook DA, Hatala R. Got power? A systematic review of sample size adequacy in health professions education research. Adv Health Sci Educ Theory Pract. 2015;20:73-83.

30. Monsieurs KG, Nolan JP, Bossaert LL, Greif R, Maconochie IK, Nikolaou NI, et al. European resuscitation council guidelines for resuscitation 2015: section 1. Executive summary. Resuscitation. 2015:1-80.

\section{Ready to submit your research? Choose BMC and benefit from:}

- fast, convenient online submission

- thorough peer review by experienced researchers in your field

- rapid publication on acceptance

- support for research data, including large and complex data types

- gold Open Access which fosters wider collaboration and increased citations

- maximum visibility for your research: over $100 \mathrm{M}$ website views per year

At BMC, research is always in progress.

Learn more biomedcentral.com/submissions 